\title{
Conocimiento objetivo y subjetivo sobre el VIH/SIDA como predictor del uso de condón en adolescentes
}

Alberto Villaseñor-Sierra, $\operatorname{Dr} C M_{1}{ }^{(1)}$ Ramiro Caballero-H oyos, $\mathrm{Dr}$ en $\mathrm{CS}_{1}{ }^{(2)}$

Alfredo Hidalgo-San Martín, MSP, ${ }^{(2)}$ José Ignacio Santos-Preciado, D r CM. ${ }^{(3,4)}$

\section{Villaseñor-Sierra A, Caballero-Hoyos R, Hidalgo-San Martín A, Santos-Preciado J. Conocimiento objetivo y subjetivo sobre el VIH/SIDA como predictor del uso de condón en adolescentes. Salud Publica Mex 2003;45 supl 1:S73-S80.}

\section{Resumen}

Objetivo. Evaluar la asociación de conocimientos objetivo y subjetivo sobre VIH/SIDA con el uso del condón. Material y métodos Se analizó la base de datos de una encuesta aleatoria, anónima y autoaplicada en 1410 adolescentes de cuatro estratos so cioeconómicos de G uadalajara, JalisCo, México, entre 1995 y 1996. El conocimiento objetivo se evaluó mediante 24 preguntas sobre VIH/SIDA y el "subjetivo" con la pregunta: "¿qué tanto crees conocer sobre el SIDA ?" Las variables predicto ras del uso del condón se identificaron mediante regresión logística y cálculo de la razón de momios con IC 95\%. Resultados El nivel de conocimiento objetivo fue regular y tuvo diferencias por estratos $(p<0.001)$ favo reciendo al medio y superior $(p=0.008)$. Del conocimiento subjetivo, los de estratos alto, medio y bajo percibieron saber "algo", y los del marginado "poco". LOS varones dijeron usar más el condón (35.4\%) que las mujeres $(15.3 \%)(p<0.001)$, y los de estratos superiores $(p<$ 0.005 ). Aunque conocimiento objetivo y subjetivo se correlacionaron $(r=0.37, p<0.001)$, un mayor conocimiento subjetivo se asoció con el uso del condón $(p<0.001)$. Conclusiones Conocimiento subjetivo, pertenecer al estrato alto o medio y ser varón, predijeron el uso constante del
Villaseñor-Sierra A, Caballero-Hoyos R,

Hidalgo-San Martín A, Santos-Preciado J.

Objective and subjective knowledge on HIV/AIDS

as predictors of condom use in adolescents.

Salud Publica Mex 2003;45 suppl 1:S73-S80.

\begin{abstract}
A bstract
Objective. To evaluate the association between objective and subjective knowledge on HIV/AIDS and condom use. Material and Methods Analysis of a database from an ano nymous, self-applied, rando mized survey conducted between 1995 and 1996. Study subjects were 1410 adolescents of four socioeconomic strata from Guadalajara, Mexico. 0 bjective knowledge was assessed with 24 questions regarding HIV/AIDS, and subjective knowledge with the question "how much do you think you know about HIV/AID S?" The variables associated with condom use were identified using logistic regression analysis and by calculating odds ratios with a $95 \%$ confidence interval. $\mathbf{R e}$ sults The degree of objective knowledge was "average", differentiated by socioeconomic strata $(p<0.001)$, and was higher in adolescents from medium and high socioeconomic strata ( $p<0.008$ ). Regarding subjective knowledge, adolescents from the low, medium, and high socioeconomic strata claimed to know "a little", and the ones from the lowest stratum claimed to know "very little". Condom use was higher in males (35.4\%), and in adolescents from high socioeconomic strata $(p<0.005)$, than in females $(15.3 \%)$ $(p<0.001)$. Although there was a correlation between ob-
\end{abstract}

El presente estudio fue realizado con financiamiento de CO N ACyT (REF: 264100-5-5037-M9406).

(1) Laboratorio de Microbiología Molecular. CIBO. Instituto Mexicano del Seguro Social. Guadalajara, Jalisco, México.

(2) Unidad de Investigación Epidemiológica y en Servicios de Salud del Adolescente. G uadalajara, Jalisco, México.

(3) Centro para la Salud de la Infancia y Adolescencia. Secretaría de Salud. México, D.F., México.

(4) Departamento de Medicina Experimental. Universidad N acional Autónoma de México. México, D.F., México. 
condón. Su uso fue diferencial por género y estrato socioeconómico.

Palabras clave: adolescencia; VIH/SIDA; conocimiento; uso de condón; México jective and subjective knowledge $(r=0.37, p<0.001)$, a higher degree of subjective knowledge was associated with condom use $(p<0.001)$. Conclusions Subjective knowledge, belonging to medium and high socioeconomic strata and being male, were predictors of condom use.

Key words: adolescents; HIV/AID S; awareness; condo m use; Mexico
E 1 síndrome de inmunodeficiencia adquirida (SIDA) es la pandemia más extendida del pasado siglo y de la actualidad. En América Latina se estima que la epidemia se inició a principios del decenio de 1980 y aunque en México se diagnosticó el primer caso de SIDA en 1983, el tiempo que pasa entre la adquisición del virus de inmunodeficiencia adquirida (VIH) y el desarrollo del SIDA, de al menos dos a 10 años, hace suponer que este virus fue introducido a México en el decenio de $1970 .^{1}$

De acuerdo con datos del Registro Nacional de Casos de SIDA, la epidemia en México ha presentado tres tendencias: a) de crecimiento lento (1983-1987); b) de crecimiento exponencial (1988-1991), y c) de crecimiento amortiguado (1992-1995). ${ }^{1,2}$ Desde 1983 y hasta el 1 de enero de 1998, se habían notificado 38390 casos de SIDA en la Secretaría de Salud. Sin embargo, tomando en consideración la corrección por subregistro debido en su mayoría a un retraso en la notificación (aproximadamente 33\%), se calcula que el número real podría ser de 60000 casos. ${ }^{1,3}$ Los estados de México y Jalisco son las entidades donde hasta el 1 de enero de 1999 se reportaron $51.5 \%$ de los casos, con una tasa acumulada de ocurrencia por 100000 habitantes de 1025 en el Distrito Federal y de 816 en Jalisco. ${ }^{1}$ La mayor parte de los casos ha ocurrido en el grupo de 25 y 39 años de edad. Del total de pacientes adultos en los que se conoce la vía de infección, en más de $87 \%$ fue adquirida por contacto sexual. ${ }^{1}$

Los eventos patogénicos asociados con la historia natural de la enfermedad son cada vez mejor conocidos. Después de la adquisición del VIH, se suceden tres fases claramente definidas; a) el periodo inmediato posterior, en donde aparecen las primeras reacciones de activación del sistema inmune y las manifestaciones de un síndrome similar a la mononucleosis infecciosa; $b$ ) un periodo prolongado de silencio clínico, en el que no hay signos ni síntomas pero en el que el virus sigue duplicándose en los ganglios linfáticos, y c) una fase de destrucción del sistema inmune, rápidamente progresiva y con la aparición de los signos y síntomas del SIDA. Por lo anterior, es razonable suponer que una buena proporción de los casos reportados en adultos de 25 a 39 años de edad pudieron haber sido contagiados con el VIH durante el periodo de adolescencia. ${ }^{4-6}$

Algunos estudios realizados en Estados Unidos de América (EUA), han mostrado un incremento en la incidencia anual de adolescentes con infección por el VIH desde $1982,{ }^{7,8}$ con una tasa similar a la observada en niños o adultos. La mayor parte $(75 \%)$ ocurrió en jóvenes entre los 17 y 19 años, con predominio en varones $(80 \%)$, con una razón de $4: 1 .{ }^{9}$ Relaciones sexuales coitales no protegidas y uso compartido de jeringas para inyectarse drogas fueron los factores de riesgo más importantes. ${ }^{8}$

Diversos informes sobre la actividad sexual en adolescentes de EUA ${ }^{10}$ y Europa, ${ }^{11}$ muestran un incremento en su actividad sexual coital, un inicio a edades más tempranas y un mayor número de parejas sexuales. Aunque en México parece haber un incremento similar, ${ }^{12}$ la proporción de adolescentes con actividad sexual coital es variable en estudiantes que inician bachillerato y la universidad, para ambas situaciones los datos son los siguientes: mujeres $5 \mathrm{y}$ $20 \%$, respectivamente y varones 17 y $50 \%$, respectivamente. Asimismo, para las mismas circunstancias, es variable la proporción de adolescentes que dijeron usar el condón en su primera relación sexual (35.5$42.2 \%$ respectivamente). ${ }^{13}$ Otro estudio realizado en jóvenes del área metropolitana de la Ciudad de México, encontró una menor frecuencia (12.3\%) en el uso de condón. ${ }^{14}$ En Guadalajara, una encuesta hecha en jóvenes de 17 a 25 años de la Universidad del estado, encontró $46 \%$ con actividad sexual coital y $15 \%$ que utilizaron en alguna ocasión el condón. ${ }^{15}$ Los resultados preliminares de la Encuesta Nacional de Juventud 2000, que incluyó una muestra de jóvenes de 12 a 29 años de edad, describen a $55 \%$ con actividad sexual coital, de los cuales $65 \%$ dijo utilizar el condón como método de protección contra las infecciones de transmisión sexual (ITS) y la adquisición del VIH. ${ }^{16}$ Además, 
$68 \%$ tuvieron su primera relación sexual entre los 15 y 19 años de edad.

El incremento en el número de casos de VIH/SIDA en México, dio lugar al establecimiento, en 1988, del Consejo Nacional para la Prevención y el Control del SIDA (CONASIDA). Una de sus metas para el año 2000 fue la de incrementar el uso del condón en al menos 30\%, y uno de sus logros, la creación de programas de prevención para grupos vulnerables como el de adolescentes. ${ }^{3}$

Además de la prevención de la adquisición del VIH mediante el uso del condón, mucho se ha escrito en relación con el efecto del grado de conocimientos sobre el VIH/SIDA (conocimiento "objetivo", medido mediante escalas de preguntas estructuradas) sobre las prácticas sexuales de riesgo, y su utilización como parte de la estrategia de prevención o modificación de dichas prácticas en adolescentes. Mientras que algunos estudios han encontrado una asociación entre un grado alto de conocimiento sobre el VIH/ SIDA y el uso de condón, o una actividad sexual coital de menor riesgo, ${ }^{17,18}$ otros señalan una relación en jóvenes con alto grado de conocimientos objetivos, asociados con actividad coital de alto riesgo para adquirir el VIH. $11,17,19$

Otros estudios han mostrado que los siguientes son predictores importantes del uso, o la intención de uso del condón: a) actitudes positivas para discutir sobre "sexo seguro" $;{ }^{20}$ b) alta percepción de "autoeficacia" en relación con las teorías de creencias personales, o portar condones, ${ }^{21} \mathrm{c}$ ) actitudes y normas subjetivas basadas en la teoría de acción razonada;,22,23 d) creencias positivas hacia el mismo y ser consciente del riesgo de la transmisión del VIH y de la utilidad de su uso para prevenirla, ${ }^{17,24-26}$ y e) género masculino, monogamia, menor edad y mayor conocimiento sobre la transmisión sexual del VIH. ${ }^{14,25,26}$ Por otra parte, algunos predictores del no uso del condón son a) incapacidad para comprarlos, ${ }^{24} \mathrm{y}$ b) tener poca confianza en su capacidad para proteger de la infección por el $\mathrm{VIH} .^{27}$

A diferencia de los resultados poco claros e inconsistentes del grado de conocimiento "objetivo" como predictor de la intención o uso del condón, la evaluación del conocimiento "subjetivo" o autopercepción del grado de conocimientos sobre el VIH/ SIDA, descrita por Phillips, ${ }^{28}$ parece brindar una herramienta de incalculable valor para identificar y predecir la intención de personas y realizar acciones concretas, tales como la aceptación para que se les haga la prueba del VIH. De la misma manera, el conocimiento subjetivo sobre el VIH/SIDA podría ser evaluado como predictor del uso del condón en adolescentes.
Los objetivos del presente estudio fueron a) describir el grado de conocimientos objetivo y subjetivo sobre el VIH/SIDA y el grado de correlación entre ellos, y b) conocer la capacidad de predicción del conocimiento objetivo y subjetivo sobre el uso constante del condón, en una muestra representativa de adolescentes de 15 a 19 años de los municipios de Guadalajara y Zapopan, Jalisco, México, y de cuatro estratos socioeconómicos.

\section{Material y métodos}

Se analizó la información vertida en una base de datos de una encuesta aplicada en hogares, con una muestra representativa de 1410 adolescentes de los municipios de Guadalajara y Zapopan, de cuatro estratos socioeconómicos durante el periodo de julio de 1995 a marzo de $1996 .{ }^{29}$ De acuerdo con el Censo General de Población y Vivienda de 1990, ${ }^{30}$ en Guadalajara habían 346648 adolescentes de 15 a 19 años de edad, en sus cuatro municipios (Guadalajara, Zapopan, Tlaquepaque y Tonalá). De estos, 286062 (82.52\%) se encontraban en los municipios de Guadalajara y Zapopan.

El cuestionario empleado -confidencial, anónimo, autoadministrado y validado- fue adaptado del cuestionario sobre conocimientos, actitudes, creencias y prácticas del programa sobre SIDA de la Organización Mundial de la Salud (OMS). ${ }^{31}$

Para la selección de la muestra, se empleó un método polietápico, tomando en consideración: a) una delimitación de conglomerados en la ubicación de Areas Geoestadísticas Básicas (AGEBS) en los municipios de Guadalajara y Zapopan; ${ }^{32}$ b) dicha delimitación permitió ubicar los estratos socioeconómicos alto, medio, bajo y marginado, de acuerdo con los indicadores físicos de vivienda y su entorno, validados por el Instituto Nacional de Estadística, Geografía e Informática (INEGI) para la zona metropolitana de Guadalajara; ${ }^{32}$ c) la selección aleatoria simple de barrios, viviendas y sujetos a entrevistar por edad. La afijación de la muestra fue proporcional, según el peso relativo de la población en cada estrato socioeconómico.

Fueron incluidos en la muestra, varones y mujeres adolescentes de 15 a 19 años de edad. Previo a la entrevista, se obtuvo el consentimiento informado por escrito de los padres o tutores $\mathrm{y}$, a su vez, la participación de los adolescentes se condicionó a ser voluntaria. No fueron incluidos aquellos adolescentes cuyos padres o tutores refirieron la existencia de trastornos mentales, convulsivos o auditivos graves o retardo mental.

La sección sobre conocimientos objetivos sobre el VIH/SIDA incluyó 24 preguntas sobre la causa, trans- 
misión y prevención del SIDA (valor de confiabilidad interna $\alpha$ de Cronbach $=0.80) .{ }^{29}$ Para su análisis en este estudio, se dio un valor ordinal de tres rangos de acuerdo con el número de respuestas codificadas como correctas: a) alto $=19$ a 24 respuestas correctas; b) regular $=12$ a $18 ;$ y c) bajo $=1$ a 11 .

El conocimiento subjetivo fue evaluado mediante una pregunta ordinal, propuesta por Phillips: ${ }^{28}$ "iqué tanto crees saber sobre el VIH/SIDA?, con las siguientes opciones de respuestas: "mucho", "algo", "un poco" y "nada".

El análisis fue descriptivo-comparativo por estratos socioeconómicos (frecuencias, medias, $\chi^{2}$, t-test, ANOVA), correlacional y con cálculo de riesgo mediante regresión logística, cálculo de razón de momios (RM) cruda y ajustada por variables de control, e Intervalos de Confianza a 95\% (IC 95\%); la variable dependiente fue el uso constante del condón; la variable exposición fue el conocimiento subjetivo, y las variables de confusión fueron el conocimiento objetivo, el estrato socioeconómico y el género. La bondad de ajuste del modelo, para predecir las variables relacionadas con el uso constante del condón, fue evaluada con la prueba de Hosmer-Lemeshow, ${ }^{33}$ para datos reagrupados y ordenados según sus probabilidades de predicción. La información fue vertida en una base de datos (FoxPro) y el análisis se hizo con el paquete estadístico Stata v.6.0.

\section{Resultados}

Información sociodemográfica. De los 1410 adolescentes, $711(50.4 \%)$ fueron varones. El $11.2 \%$ pertenecieron al estrato alto, $40.4 \%$ al medio; $30.2 \%$ al bajo y $18.2 \%$ al marginado. La proporción de adolescentes por grupos de edad en años cumplidos fue como sigue: 15 $(20.5 \%), 16(20.1 \%) 17(19.9 \%), 18(20.4 \%)$, y $19(19.2 \%)$. El 50.6\% de la muestra se conformó por estudiantes de tiempo completo; $21.4 \%$ trabajaban; $13.5 \%$ trabajaban y estudiaban; $13.9 \%$ no estudiaban ni trabajaban y $0.5 \%$ tenían otras actividades. El nivel escolar fue como sigue; analfabetas $0.9 \%$, primaria $11.3 \%$, secundaria $34.3 \%$, carrera técnica $5.8 \%$, preparatoria y más $47.6 \%$.

Conocimiento objetivo sobre VIH/SIDA. El número promedio de respuestas correctas en relación con conocimientos generales fue 13.3 y 12.7 , de 24 posibles en varones y mujeres respectivamente $(t=121.6, p<0.001)$. El número de aciertos en adolescentes de los estratos alto, medio, bajo y marginado descendió de 14.8 en el alto a 11.9 en el marginado (cuadro I). Hubo diferencias significativas entre el número de aciertos en adolescentes de los estratos alto y medio en relación con los del medio y marginado (ANOVA: $F=19.39, \mathrm{gl} 3$; $p<0.001)$. No hubo una diferencia significativa entre el promedio de aciertos en adolescentes del estrato bajo y el marginado.

\section{Cuadro I \\ Grado de conocimientos objetivo y SUbjetivo sobre el VIH/SIDA en adolescentes por estratos socioeconómicos. Guadalajara, MéXico, 1995-1996}

\begin{tabular}{|c|c|c|c|c|c|c|c|c|}
\hline \multirow[b]{3}{*}{ Conocimiento objetivo* } & \multicolumn{8}{|c|}{ Estrato socioeconómico } \\
\hline & \multicolumn{2}{|c|}{$\begin{array}{c}\text { Alto } \\
(n=158)\end{array}$} & \multicolumn{2}{|c|}{$\begin{array}{c}\text { Medio } \\
(n=569)\end{array}$} & \multicolumn{2}{|c|}{$\begin{array}{c}\text { Bajo } \\
(n=424)\end{array}$} & \multicolumn{2}{|c|}{$\begin{array}{l}\text { Marginado } \\
(\mathrm{n}=257)\end{array}$} \\
\hline & $\begin{array}{c}\mathrm{V}(79) \\
(\%)\end{array}$ & $\begin{array}{c}M(79) \\
(\%)\end{array}$ & $\begin{array}{c}(286) \\
(\%)\end{array}$ & $\begin{array}{c}M(283) \\
(\%)\end{array}$ & $\begin{array}{c}\overline{V(215)} \\
(\%)\end{array}$ & $\begin{array}{c}M(209) \\
(\%)\end{array}$ & $\begin{array}{c}\bar{V}(130) \\
(\%)\end{array}$ & $\begin{array}{c}M(127) \\
(\%)\end{array}$ \\
\hline Bajo (1-11) & 0.0 & 2.5 & 3.9 & 5.3 & 9.8 & 9.6 & 9.2 & 14.2 \\
\hline Regular (12-18) & 81.0 & 88.6 & 88.1 & 87.3 & 82.8 & 86.6 & 84.6 & 82.7 \\
\hline Alto (19-24) & 19.0 & 8.9 & 8.0 & 7.4 & 7.4 & 3.8 & 6.2 & 3.1 \\
\hline Conocimiento subjetivo ${ }^{\ddagger}$ & $V(n=79)$ & $M(n=79)$ & $V(n=286)$ & $M(n=284)$ & $V(n=216)$ & $M(n=209)$ & $V(n=130)$ & $M(n=127)$ \\
\hline $\mathrm{N}$ ada y poco§ & 20.3 & 17.7 & 30 & 36.6 & 39.8 & 48.3 & 43 & 42 \\
\hline Algo & 57 & 75.9 & 61.2 & 57 & 55.1 & 48.8 & 54.6 & 36.2 \\
\hline Mucho & 22.8 & 6.3 & 8.7 & 6.3 & 5.1 & 2.9 & 2.3 & 4.7 \\
\hline \multicolumn{9}{|c|}{$\begin{array}{l}\text { * Varones } \chi^{2}=21.0 \text {, d.f. } 6 ; p<0.005 \text {, mujeres } \chi^{2}=34.3 \text {, d.f. } 6, p<0.001 \\
\text { ₹ Varones } \chi^{2}=41.2 \text {, d.f. } 6 ; p<0.001 \text {, mujeres } \chi^{2}=43.2 \text {, d.f. } 6 ; p<0.001 \\
\text { \& Para efecto del cálculo de la } \chi^{2} \text { se juntaron las categorías originales }\end{array}$} \\
\hline \multicolumn{9}{|c|}{$\begin{array}{l}\text { Nota: coeficiente de correlación rho de Spearman para conocimiento objetivo y subjetivo }=0.37, p<0.001 \\
n=1410 \text { en porcentajes }\end{array}$} \\
\hline
\end{tabular}


Conocimiento subjetivo sobre VIH y SIDA. En los varones y mujeres del estrato marginado predominó la autopercepción de tener bajos conocimientos sobre VIH/SIDA, mientras que en los otros estratos predominó la percepción de tener un conocimiento regular. En relación con el género, la categoría de los que percibieron saber "mucho", fue predominante en varones del estrato alto, quienes presentaron una mayor proporción que las mujeres del mismo estrato (cuadro I). Correlación entre el grado de conocimiento objetivo y subjetivo. Un grado bajo (1 a 11 aciertos de 24) sobre conocimientos "objetivos" acerca de VIH/SIDA se asoció con una percepción de saber "poco" en $60.6 \%$ de los adolescentes de uno u otro género y de los cuatro estratos socioeconómicos. Sin embargo, un grado medio (12 a 18 aciertos de 24) y un grado alto (19 a 24 aciertos de 24) se asociaron con una percepción de saber "algo" en $56.7 \%$ y en $66.7 \%$ de los adolescentes, respectivamente. Hubo una asociación moderada y significativa entre el grado de conocimiento objetivo y subjetivo (Coeficiente de correlación rho de Spearman $=0.37, p<$ 0.001).

Prácticas sexuales. La proporción de adolescentes que dijeron tener relaciones sexuales coitales fue mayor entre varones $(27 \%)$ que entre mujeres $(8.4 \%)(p<0.001)$. No hubo una diferencia significativa entre la proporción de quienes dijeron tener relaciones sexuales coitales, en los distintos estratos socioeconómicos.

Uso constante del condón. El uso constante del condón ("siempre") fue mayor en varones (35.4\%) que en las mujeres, quienes reportaron el uso del condón por su pareja $(15.3 \%)(p<0.001)$. En los varones, una mayor frecuencia en el uso constante del condón se asoció en forma directa con el estrato socioeconómico $\left(\chi^{2}=16.8\right.$, $p=0.001$ ), mientras que en las mujeres, el uso constante del condón por sus parejas presentó un comportamiento bimodal, con una mayor frecuencia en los estratos medio y marginado. Considerando a adolescentes de uno u otro género, hubo una mayor frecuencia en el uso constante del condón en estratos socioeconómicos alto y medio que en el bajo y marginado $\left(\chi^{2}=13.2 \mathrm{gl}(v) 3 ; p<0.005\right)$.

El análisis de regresión logística mostró que individualmente, los grados más altos de conocimiento objetivo $(R M=2.8$, IC $95 \% 1.1-6.7)$ y subjetivo $(R M=$ 2.6, IC 95\% 1.4-4.8) predicen un mayor uso constante de condón. Ajustada por las variables confusoras, la variable de exposición (el mayor conocimiento subjetivo), mantuvo su significancia como factor predictor $(R M=2.3$, IC $95 \%$ 1.2-4.5) del uso constante del condón. Tanto en el análisis de razón de momios crudas como ajustadas, las variables confusoras, estratos socioeconómicos, alto y medio, y el género masculino tuvieron un mayor peso predictivo que el factor de conocimientos (cuadro II). La bondad de ajuste del modelo para predecir las variables relacionadas con el uso constante del condón fue adecuada, debido a que la prueba de Hosmer y Lemeshow mostró no ser significativa $\left(\chi^{2}=20.3, \mathrm{gl}=17 ; p=0.26\right)$.

\section{Discusión}

El riesgo de infección por el VIH durante la adolescencia es un problema creciente, ${ }^{7,9}$ debido al incremento en la proporción de jóvenes con relaciones sexuales coitales, a la edad de inicio más temprano, a la inconsistencia o el poco uso del condón, y al inicio del uso de drogas ilegales. ${ }^{10,12,14,16,34}$ Este incremento en el riesgo podría estar asociado con una autopercepción de bajo riesgo, sentido de invulnerabilidad, una mayor exposición a relaciones coitales riesgosas; todo esto, a su vez favorecido por la ausencia de manifestaciones clínicas durante el periodo que transcurre desde la infección hasta el desarrollo de la enfermedad clínica del SIDA. ${ }^{4,5}$

Las campañas de prevención del SIDA han procurado incrementar el nivel de conocimiento objetivo sobre las formas de adquisición y transmisión del VIH, así como de las manifestaciones clínicas del SIDA y su prevención mediante la práctica de la abstinencia, la fidelidad de pareja o el uso del condón en aquellos con relaciones sexuales coitales. Sin embargo, diversos estudios sobre el grado de conocimiento objetivo ${ }^{17,35-37} \mathrm{y}$ su impacto en las prácticas sexuales de adolescentes de diferentes poblaciones, han mostrado una variabilidad e inconsistencia considerables. Cohortes de adolescentes seguidos durante 3-4 años muestran que el grado de conocimientos sobre VIH/SIDA puede fluctuar en cada individuo de mayor a menor y viceversa. ${ }^{38}$ Algunos estudios muestran un alto grado de conocimientos asociados con una actividad sexual coital de bajo riesgo, o que se modifica de una de mayor a otra de menor riesgo, ${ }_{1}^{17}$ mientras que en otros los conocimientos se asocian con una actividad sexual de alto riesgo. ${ }^{19}$

En el presente estudio, el grado promedio de conocimiento objetivo en los adolescentes fue regular, pero diferencial por estrato socioeconómico (mayor en los estratos alto y medio) y género (mayor en varones). Otros estudios han reportado también un mayor grado de conocimientos en adolescentes varones y de un estrato socioeconómico alto, ${ }^{39}$ así como un menor grado de conocimientos asociado con grupos minoritarios o de bajo nivel de escolaridad. ${ }^{40}$ Asimismo, se ha descrito un mayor número de casos de SIDA asociado con personas con alto porcentaje de desempleo y baja es- 


\section{Cuadro II \\ Conocimientos subjetivo Y objetivo como factores PREDictores del uso CONSTANTE del CONDón en adolescentes de Guadalajara, México. 1995-1996}

\begin{tabular}{|c|c|c|c|c|c|c|}
\hline \multirow[b]{2}{*}{ Factores predictores } & \multicolumn{2}{|c|}{ Uso constante del condón } & \multirow{2}{*}{$\begin{array}{l}\text { Razón } \\
\text { de momios } \\
\text { cruda }\end{array}$} & \multirow[b]{2}{*}{ IC $95 \%$} & \multirow{2}{*}{$\begin{array}{l}\text { Razón } \\
\text { de momios } \\
\text { ajustada* }\end{array}$} & \multirow[b]{2}{*}{ IC $95 \%$} \\
\hline & $\begin{array}{c}\text { Sí } \\
\mathrm{N}=77\end{array}$ & $\begin{array}{c}\text { No } \\
N=174\end{array}$ & & & & \\
\hline \multicolumn{7}{|c|}{ Conocimiento subjetivo } \\
\hline $\mathrm{N}$ ada-poco & 16 & 70 & 1 & & 1 & \\
\hline Algo-mucho & 61 & 104 & 2.6 & $1.4-4.8$ & 2.3 & $1.2-4.5$ \\
\hline \multicolumn{7}{|c|}{ Conocimiento objetivo sobre VIH/SIDA } \\
\hline Bajo-regular & 65 & 164 & 1 & & 1 & \\
\hline Alto & 11 & 10 & 2.8 & $1.1-6.7$ & 2.4 & $0.9-6.6$ \\
\hline \multicolumn{7}{|c|}{ Estrato socioeconómico } \\
\hline Alto & 13 & 112 & 6.3 & $1.9-20.8$ & 6.5 & $1.8-23.6$ \\
\hline Medio & 42 & 75 & 3.2 & $1.2-8.7$ & 3.8 & 1.3-11.1 \\
\hline Bajo & 17 & 58 & 1.7 & $0.6-4.9$ & 2.0 & $0.6-6.3$ \\
\hline Marginado & 5 & 29 & 1 & & 1 & \\
\hline \multicolumn{7}{|l|}{ Género } \\
\hline Varones & 68 & 124 & 3.04 & $1.4-6.5$ & 3.4 & $1.5-7.6$ \\
\hline Mujeres & 9 & 50 & 1 & & 1 & \\
\hline
\end{tabular}

Hosmer y Lemeshow goodness-of-fit test: chi-square $=20.3, \mathrm{gl}=17 ; p=0.26$

$n=257$ en frecuencias, razón de momios cruda y ajustada e intervalos de confianza de $95 \%$

colaridad. ${ }^{41}$ Asumiendo que el origen del conocimiento sobre el VIH/SIDA en nuestros adolescentes es producto de las campañas de prevención sobre el SIDA, su impacto parece modesto y diferencial en favor de los jóvenes de estratos socioeconómicos alto y medio. Otros factores que podrían influir en esta resultante es la limitada capacidad económica y cultural de la mayoría de los adolescentes para disponer o procurar de medios impresos informativos (periódico, revistas), o para lograr un nivel adecuado de escolaridad. Además, las características de nuestra sociedad parece que aún favorecen un mayor desarrollo de los varones.

En este estudio, aunque un grado alto de conocimiento objetivo (no ajustado por variables de confusión) predijo el uso constante del condón en aquellos adolescentes con actividad sexual coital, este uso llegó a niveles no significativos al ajustarlo, indicando su pobre asociación con las prácticas sexuales.

En relación con el conocimiento subjetivo, la percepción predominante y homogénea en adolescentes de todos los estratos socioeconómicos y de uno u otro género fue la de saber "algo" sobre el VIH/SIDA. Esto contrasta con el conocimiento objetivo, altamente diferencial por estrato y género. Lo anterior podría indicar que los mensajes de prevención sobre VIH/SIDA no son captados cognoscitivamente pero sí de modo "perceptivo" o conceptual. Por otra parte, y a diferencia del conocimiento objetivo, el conocimiento subjetivo fue un predictor significativo del uso constante del condón aun después de ser ajustado por variables confusoras. Aunque de manera intuitiva pareciera que la asociación podría ir del conocimiento (subjetivo) al comportamiento (uso del condón), las características del diseño del estudio (transversal), no permiten aseverar la dirección de dicha asociación.

Con relación al conocimiento subjetivo, es interesante la semejanza entre el estudio descrito por Phillips $^{28}$ en el que un mayor conocimiento subjetivo predijo la intención de aceptar hacerse la prueba del VIH (de suma importancia en la epidemiología del SIDA), y el presente en cuanto a que el conocimiento subjetivo fue capaz de predecir el uso constante del 
condón (de mayor importancia para la prevención de la infección por VIH en quienes tienen actividad sexual coital).

Estos hallazgos nos deben llevar a explorar y profundizar en los elementos que integran el conocimiento "subjetivo", así como su integración a las campañas de prevención del VIH/SIDA en las que ya se ha contemplado: a) la promoción, la abstinencia sexual o retraso en su inicio hasta conseguir una pareja estable, b) ofrecimiento de entrenamiento en técnicas y habilidades para evitar prácticas de alto riesgo, c) apoyo de servicios de salud, d) mensajes para incrementar la autopercepción de riesgo, y e) disponibilidad de condones gratuitos y ejercicio de sexo seguro. La adición de elementos que impacten la percepción subjetiva a los actuales mensajes preventivos, podría dar como resultado una mayor reducción de prácticas de alto riesgo y un incremento en el uso del condón. 22,43,44

En relación con la proporción de adolescentes varones $(27 \%)$ y mujeres $(8.4 \%)$ con actividad sexual coital y con el uso "consistente" del condón (35.4\% y $15.3 \%$, respectivamente) en el presente estudio, debemos insistir en que sólo fueron analizados los datos de aquellos adolescentes que dijeron usar "siempre" el condón. Por esto, la proporción descrita parecería muy por debajo de lo referido en la Encuesta Nacional de la Juventud $2000^{16}$ para actividad sexual coital $(55 \%)$ y uso de condón (65\%). Sin embargo, debe tomarse en cuenta que esta última incluyó a jóvenes de 12 a 29 años de edad (muchos casados) y sólo preguntaron la frecuencia con que usaban condón para evitar el contagio de VIH e ITS, por lo que no es comparable. En la encuesta realizada por Micher en estudiantes de 15 a 19 años que ingresaron a bachillerato o a la facultad en la Universidad Nacional Autónoma de México (UNAM) ${ }^{13}$ tuvieron una actividad sexual coital promedio en varones $33.5 \%$ y mujeres $12.5 \%$, muy similar a lo descrito en nuestro estudio; y un uso de condón "en su primera relación" de 42.2 y $35.5 \%$, respectivamente, por encima de lo encontrado por nuestro grupo. En este estudio de la UNAM tampoco consideraron un criterio tan estricto del uso de condón como el nuestro. Finalmente, la proporción de actividad sexual coital y uso "en alguna ocasión" del condón, descrita por Tello en Guadalajara (46 y 15\% respectivamente), ${ }_{1}^{15}$ aunque fue casi el doble en cuanto a actividad sexual coital y similar en uso de condón, se investigó en un grupo no comparable de jóvenes de 17 a 25 años de edad.

En conclusión, el presente estudio muestra que el grado de conocimiento "subjetivo" sobre el VIH/SIDA predice el uso consistente del condón en adolescentes de ambos géneros y de todos los estratos socio- económicos. En contraste, el conocimiento objetivo fue diferencial por género y por estrato socioeconómico y no se asoció con el uso consistente del condón. Por ello, los mensajes para la prevención de la infección del VIH/SIDA en este grupo altamente vulnerable podrían enriquecerse al incluir aspectos que puedan impactar en el área del conocimiento subjetivo.

Al comparar las proporciones de adolescentes del presente estudio, varones y mujeres con actividad sexual coital, con los de otros realizados recientemente en nuestro país, nos da la impresión que va en aumento. Sin embargo, el uso "consistente" del condón fue bajo en nuestro estudio y difícil de comparar, debido a la carencia de estudios que incluyan a poblaciones representativas similares. Esto podría traducir un riesgo mayor para la adquisición del VIH, así como la necesidad de incrementar las campañas de prevención.

Para finalizar, debe mencionarse que el estudio tiene por lo menos dos limitaciones que deben considerarse al evaluar sus resultados. Por una parte, los datos provienen de una encuesta por muestreo que recolectó autorreportes de prácticas sexuales y de uso de condón. Al ser una recolección de medidas indirectas, estuvo sujeta a algunas imprecisiones basadas en la honestidad de la respuesta y en la comprensión de las preguntas. Por otra parte, la pregunta sobre el uso de condón recolectó frecuencias de uso en las categorías siempre, a veces y nunca. No indagó sobre algunas situaciones que relacionadas con el uso constante del condón influyen sobre el grado de riesgo de infección y la eficacia del condón. De Visser y Smith, ${ }^{44}$ por ejemplo, encontraron en estudiantes australianos con vida sexual activa que es frecuente la penetración sin protección, colocándose el condón momentos antes de la eyaculación. De esa manera, el riesgo de contagio del VIH por el intercambio de secreciones sexuales estaría presente y la acción del uso del condón quedaría como un acto irrelevante para su protección.

\section{Referencias}

1. Magis-Rodríguez C, D el Río-Chiriboga C. Epidemiología del VIH y del SIDA en México. En: Ponce de León RS, Rangel FMS, ed. SIDA. Aspectos clínicos y terapéuticos. México, D.F. : McG raw-Hill Interamericana, 2000: 1-10.

2. Valdespino-Gómez JL, García-García ML, Del Río-Zolezzi A, Loo-Méndez E, Magis-Rodríguez C, Salcedo-Alvarez RA. Epidemiología del SIDA/ VIH en México; de 1983 a marzo de 1995. Salud Publica Mex 1995;37: 556-571.

3. Magis-Rodríguez C, Bravo-G arcía E,Anaya-López L, U ribe-Zúniiga P. La situación del SIDA en México a finales de 1998. Enf Infecc Microbiol 1998;18:236-244. 
4. Medley GF,Anderson RM, C ox DR, Billard L. Incubation period of AIDS in patients infected via blood transfusion. N ature 1987;328:719-721. 5. Lui KJ, Darrow W W, Rutherford GW 3d.A model-based estimate of the mean incubation period for AIDS in homosexual men. Science 1988; 240:1333-1335.

6. Camacho ME. Evolución natural de la infección por VIH. En: Ponce de León RS, Rangel FMS, ed. SIDA aspectos clínicos y terapéuticos. México, D.F. : McG raw-Hill Interamericana, 2000:115-138.

7. Center for D isease Control and Prevention.AIDS and human immunodeficiency virus infection in the United States: 1988 update. Morb Mortal W kly Rep 1989;38:1-38.

8. Bowler S, Sheon AR, D 'Angelo LJ, Vermund SH. HIV and AIDS among adolescents in the United States: Increasing risk in the 1990s. J Adolesc 1992;15:345-371

9. Manoff SB, Gayle HD, Mays MA, Rogers MF.Acquired immunodeficiency syndrome in adolescents: Epidemiology, prevention and public health issues. Pediatr Infect D is J 1989;8:309-314.

10. H offerth SL, Kahn JR, Baldwin W. Premarital sexual activity among U.S. teenage women over the past three decades. Fam Plann Perspect 1987; 19:46-53.

11. Klanger B,Tyden T, Ruusuvaara L. Sexual behavior among adolescents in Uppsala, Sweden. J A dolesc Health 1993;14:468-474.

12. Consejo $\mathrm{N}$ acional de Población. Encuesta $\mathrm{N}$ acional sobre sexualidad y familia en jóvenes de educación media superior.; México, D.F.: Conapo, 1988.

13. Micher CJM, Silva BJS. N ivel de conocimientos y prácticas de riesgo para enfermedades de transmisión sexual (ETS) en alumnos de primer ingreso a la Universidad N acional Autónoma de México, UNAM. SIDAETS 1997;3:68-73.

14. García-Baltazar J, Figueroa-Perea JG. Práctica anticonceptiva en adolescentes jóvenes del área metropolitana de la ciudad de México. Salud Publica Mex 1992;34:413-426.

15. Tello A. Los jóvenes, el sector más vulnerable ante el SIDA. Gac Universitaria 2001;1(223): 14-15.

16. Pérez IJA. Encuesta N acional de Juventud 2000. Resultados Preliminares; México, D.F.: Secretaría de Educación Pública, 2001: 1.

17. Siegel D, Lazarus N , Krasnovsky F, D urbin M, Chesney M.AID S knowledge, attitudes, and behavior among inner city, junior high school students. J Sch Health 1991;61:160-165.

18. Rickert VI, Jay MS, Gottlieb A, Bridges C. Adolescents and AIDS. Female's attitudes and behaviors toward condom purchase and use. J Adolesc Health C are 1989;10:313-316.

19. DiC lemente RJ, Brown LK, Beausoleil NI, Lodico M. Comparison of AIDS knowledge and HIV-related sexual risk behaviors among adolescents in low and high AIDS prevalence communities. J Adolesc Health Care 1993;14:231-236.

20. Troth A, Peterson CC. Factors predicting safe-sex talk and condom use in early sexual relationships. Health Commun 2000;12:195-218.

21. Maxwell AE, Bastani R, W arda US. Knowledge and attitudes toward condom use-Do they predict behavior among Filipino Americans? Ethn $D$ is 2000;10:113-124.

22. Bandawe CR, Foster D.AIDS-related beliefs, attitudes and intentions among Malawian students in three secondary schools. AIDS Care 1996;8:223-232.

23. Klepp KI, N deki SS,T huen F, Leshabari M, SehaAM. Predictors of intention to be sexually active amongTanzanian school children. East Afr Med J 1996;73:218-224

24.Stiffman AR, Dore P, C unningham RM. Inner-city youths and condom use: $H$ ealth beliefs, clinic care, welfare, and the HIV epidemic.A dolescence 1994:29:805-820.

25. Bazargan M, Kelly EM, Stein JA, H usaini BA, Bazargan SH. C orrelates of HIV risk-taking behaviors among African-A merican college students: The effect of HIV knowledge, motivation, and behavioral skills. J N atl Med Assoc 2000;92:391-404.

26. Reitman D, St Laurence JS, Jefferson KW,A lleyne E, Brasfield TL, Shirley A. Predictors of African A merican adolescents' condom use and HIV risk behavior. AIDS Educ Prev 1996;8:499-515.

27. Stiffman AR, Earls F, D ore P, C unningham RM. Changes in acquired immunodeficiency syndrome-related risk behavior after adolescence: Relationships to knowledge and experience concerning human immunodeficiency virus infection. Pediatrics 1992:89:950-956.

28. Phillips KA. Subjective knowledge of AIDS and use of HIV testing.Am J Public Health 1993;83:1460-1462.

29. Caballero-Hoyos R, Villaseñor-Sierra A, San Martín AH. Fuentes de información y su relación con el grado de conocimientos sobre SIDA en adolescentes de México. Rev Saude Publica 1997;31:351-359.

30. Instituto Nacional de Estadística, Geografía e Informática. XI Censo $N$ acional de Población y Vivienda, 1990. Resultados definitivos. D atos por AGEB urbana. México, D.F.: IN EGI, 1990.

31. W orld Health 0 rganization. Research package: Knowledge, attitudes, beliefs and practices on AIDS survey (KABP). Appendix: $Q$ uestionnaire. W ashington D.C.:W ho, 1990.

32. Instituto $\mathrm{N}$ acional de Estadística, Geografia e Informática. Estratificación socio-económica de la zona Metropolitana de Guadalajara. 1992; Guadalajara, Jalisco. IN EGI, 1992

33. Hosmer DW, Lemeshow S. Applied logistic regression. N ueva York (N Y): John W iley \& Sons, 1989.

34. Caballero-H oyos R, Madrigal DLE, Hidalgo SMA,Villaseñor A. El Consumo de tabaco, alcohol y drogas ilegales, en los adolescentes de diferentes estratos socioeconómicos de Guadalajara. Salud Mental 1999;22:1-8. 35. Skurnick JH, Johnson RL, Q uinones MA, Foster JD, Louria DB. N ew Jersey high school students' knowledge, attitudes, and behavior regarding AIDS.AID S Educ Prev 1991;3:21-30.

36. Koopman C, Rotherman-Borus MJ, Henderson R, Bradley JS, Hunter J. Assessment of knowledge of AIDS and beliefs about AIDS prevention among adolescents. AIDS Educ Prev 1990;2:58-69.

37. DiC lemente RJ,Zorn J,Temoshok L.A dolescents and AID S:A survey of knowledge, attitudes and beliefs about AIDS in San Francisco. A m J Public Health 1986;76:1443-1445.

38. Zimet GD, D iC lemente RJ, Lazebnik R,Anglin TM, Ellick EM,W illiams P. Changes in adolescents' knowledge and attitudes about AIDS over the course of the AIDS epidemic. J Adolesc Health 1993;14:85-90.

39. Yelibi S, Valenti P, Volpe C, Caprara A, Dedy S, Tape G. Sociocultural aspects of AIDS in an urban peripheral area of Abidjan. AIDS $C$ are 1993:5:187-197.

40. DuRant RH,A shworth CS, N ewman CL, MCG ill L, Rabun C, Baranowski T.AIDS/HIV knowledge level and perceived chance of having HIV among rural adolescents. J Adolesc Health 1992;13:499-505.

41. Díaz T, C hu SY, Buehler JW, Boyd D, C heco PJ, C onti L et al. Socioeconomic differences among people with AID S: Results from a multistate surveillance project.Am J Prev Med 1994;10:217-222.

42. St Laurence JS, Jefferson KW, Alleyne E, Brasfield TL. Comparison of education versus behavioral skills training interventions in lowering sexual HIV-risk behavior of substance-dependent adolescents. J Consult Clin Psychol 1995;63:154-157.

43. Rotherman-Borus MJ, Koopman C, Haignere C, D avies M. Reducing HIV sexual risk behaviors among runaway adolescents. JAMA 1991;266:1237-1241.

44. De Visser RO, Smith AMA.W hen always isn't enough. Implications of the late aplications of condoms for the validity of self-reported condom use. AIDS C are 2000;12:221-226. 